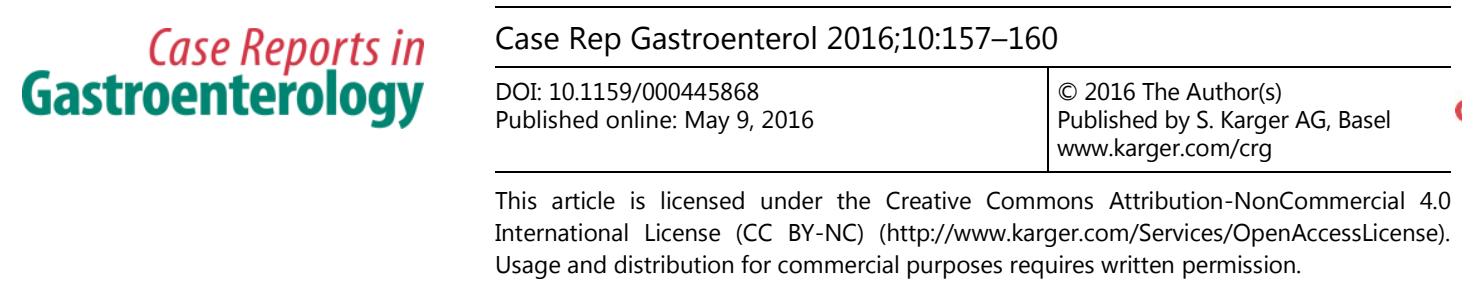

\title{
Efficacy of Combined Mesalazine Plus Corticosteroid Enemas for Diversion Colitis after Subtotal Colectomy for Ulcerative Colitis
}

\author{
Satohiro Matsumoto Hirosato Mashima \\ Department of Gastroenterology, Saitama Medical Center, Jichi Medical University, \\ Omiya, Saitama, Japan
}

\section{Keywords}

Diversion colitis · Ulcerative colitis · Combination enema · Mesalazine enema

\begin{abstract}
Diversion colitis is a benign inflammatory process that occurs in any part of the large bowel excluded from the fecal stream by a diverting colostomy. While most of the patients with diversion colitis usually are asymptomatic, a minority has abdominal pain and rectal discharge of blood or mucus. A 65-year-old Japanese man was diagnosed as having diversion colitis with ulcerative colitis at 4 months after subtotal colectomy. Corticosteroid and mesalazine enemas were started nonsynchronously. A proctoscopy after 2 months showed no response. Prednisolone injections were started at $1.0 \mathrm{mg} / \mathrm{kg}$ daily, but the mucosal inflammation still failed to improve. A combined mesalazine $1 \mathrm{~g}$ plus prednisolone sodium phosphate $20 \mathrm{mg}$ enema was started once daily. The rectal bleeding and endoscopic findings improved. Finally proctectomy and ileal pouch-anal anastomosis were successfully performed. A combined mesalazine plus corticosteroid enema may be effective in patients with diversion colitis associated with ulcerative colitis.

(C) 2016 The Author(s) Published by S. Karger AG, Basel
\end{abstract}




\section{Introduction}

Diversion colitis typically occurs in diverted segments of the colon following surgery. While patients with diversion colitis usually are asymptomatic, a minority of patients has abdominal pain and rectal discharge of blood or mucus [1]. Most patients with diversion colitis show improvement with reanastomosis within 2 weeks [2]. Effective treatments with short-chain fatty acids [3], mesalazine enemas [2, 4], and corticosteroid enemas [1] have been reported. Herein, we present a case of successful treatment of diversion colitis developing after subtotal colectomy for ulcerative colitis (UC) with combined mesalazine plus corticosteroid enemas.

\section{Case Report}

A 65-year-old Japanese man diagnosed as having UC, pancolitis-type, was started on treatment with prednisolone injections at $1.0 \mathrm{mg} / \mathrm{kg}$ daily and oral mesalazine. The symptoms failed to improve, and the patient was transferred to our hospital. On the 2nd hospital day, his abdominal pain worsened and an abdominal X-ray showed free air and distension of the entire colon. Emergent subtotal colectomy and ileostomy were performed. The resected specimen showed a markedly distended colon with diffuse ulcerations, and a perforation in the sigmoid colon.

The postoperative clinical course was uneventful and administration of mesalazine $1 \mathrm{~g}$ suppositories (Pentasa suppositories, Kyorin Pharmaceutical Co., Ltd., Japan) was started. At 4 months after the colectomy, the patient developed rectal bleeding, and proctoscopy revealed moderate mucosal inflammation in the diverted rectum (fig. 1a). A biopsy from the rectum showed ulcer, granulation tissue and epithelial defect. Stool cultures were negative for bacterial pathogens and Clostridium difficile latex antigen and toxin assay. Diversion colitis or exacerbation of UC was suspected and treatment was started with a corticosteroid enema in the morning and mesalazine enema in the evening.

A repeat proctoscopy after 2 months showed no response. Therefore, prednisolone injections were started again at $1.0 \mathrm{mg} / \mathrm{kg}$ daily; however, the mucosal inflammation still failed to improve. The only symptom was bleeding from the diverted rectum, and blood test results were within normal limits. The patient was then started on treatment with a combined mesalazine $1 \mathrm{~g}$ (Pentasa enema, Kyorin, Japan) plus prednisolone sodium phosphate 20 mg enema (Predonema, Kyorin, Japan) once daily. The rectal bleeding resolved gradually and follow-up proctoscopy after 4 weeks revealed only mild mucosal inflammation (fig. 1b). Thereafter, proctectomy and ileal pouch-anal anastomosis were performed.

\section{Discussion}

Diversion colitis is a benign inflammatory condition that can occur in any colonic segment excluded from the fecal stream by a diverting colostomy. Because this patient showed clinical exacerbation 4 months after subtotal colectomy for UC, we diagnosed him as having diversion colitis associated with UC. In this case, the diversion colitis seemed to occur with a longer residual rectum and sigmoid colon (around $20 \mathrm{~cm}$ ). Mesalazine enema is safe and effective for the treatment of diversion colitis [2, 4]. However, our patient failed to respond to nonsynchronous treatment with mesalazine and corticosteroid enemas. The efficacy of 
Matsumoto and Mashima: Efficacy of Combined Mesalazine Plus Corticosteroid Enemas for Diversion Colitis after Subtotal Colectomy for Ulcerative Colitis

combined beclomethasone plus mesalazine enemas in cases of active UC has been reported [5]. Furthermore, combined beclomethasone and mesalazine treatment appears to be clearly superior to monotherapy with either drug [6]. Therefore, we attempted treatment with combined mesalazine plus corticosteroid enemas. This provided sufficient symptomatic improvement to allow the treatment to proceed to the next step.

In conclusion, although we cannot explain why synchronous and nonsynchronous administrations varied in effectiveness, combined mesalazine plus corticosteroid enemas may be effective in patients with diversion colitis associated with UC.

\section{Statement of Ethics}

The authors have no ethical conflicts to disclose.

\section{Disclosure Statement}

The authors declare no conflicts of interest for this article.

\section{References}

1 Glotzer DJ, Glick ME, Goldman H: Proctitis and colitis following diversion of the fecal stream. Gastroenterology 1981;80:438-441.

2 Tripodi J, Gorcey S, Burakoff R: A case of diversion colitis treated with 5-aminosalicylic acid enemas. Am J Gastroenterol 1992;87:645-647.

-3 Harig JM, Soergel KH, Komorowski RA, Wood CM: Treatment of diversion colitis with short-chain-fatty acid irrigation. N Engl J Med 1989;320:23-28.

4 Triantafillidis JK, Nicolakis D, Mountaneas G, Pomonis E: Treatment of diversion colitis with 5aminosalicylic acid enemas: comparison with betamethasone enemas. Am J Gastroenterol 1991;86:1552-1553.

-5 Guslandi M, Giollo P, Testoni PA: A combination of rectal beclomethasone diproprionate and mesalazine in ulcerative proctitis. Scand J Gastroenterol 2008;43:639-640.

6 Mulder CJ, Fockens P, Meijer JW, van der Heide H, Wiltink EH, Tytgat GN: Beclomethasone dipropionate ( $3 \mathrm{mg}$ ) versus 5-aminosalicylic acid ( $2 \mathrm{~g}$ ) versus the combination of both $(3 \mathrm{mg} / 2 \mathrm{~g}$ ) as retention enemas in active ulcerative proctitis. Eur J Gastroenterol Hepatol 1996;8:549-553. 
Matsumoto and Mashima: Efficacy of Combined Mesalazine Plus Corticosteroid Enemas for Diversion Colitis after Subtotal Colectomy for Ulcerative Colitis
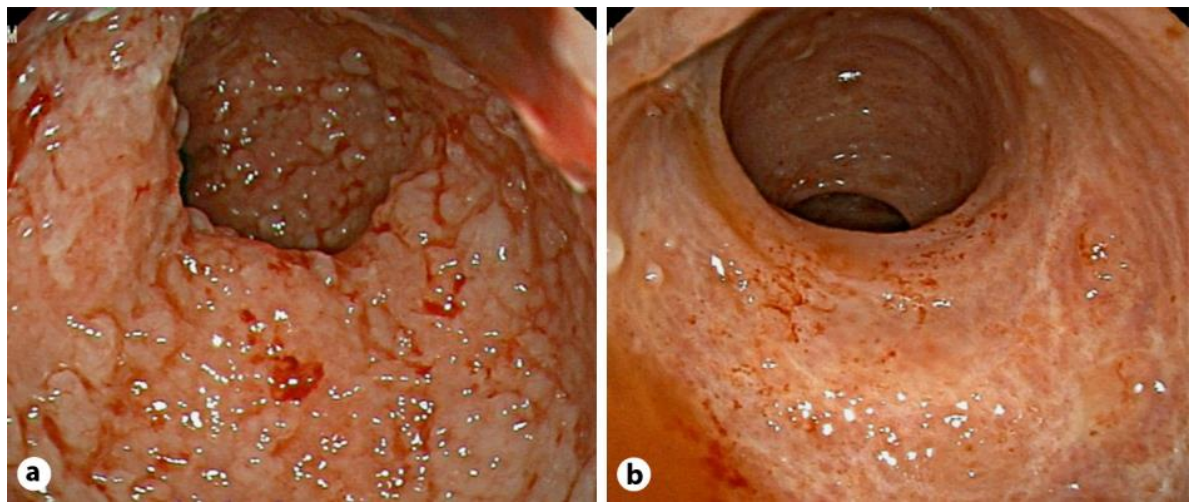

Fig. 1. Findings of the proctoscopy in the diverted rectum. a Proctoscopy at 4 months after subtotal colectomy reveals loss of the vascular pattern, erosions and contact bleeding. $\mathbf{b}$ Proctoscopy at 4 weeks after the start of the combination enema reveals mild erosions. 